\title{
Labyrinthe
}

21 | 2005 (2)

Communauté en pièces : d'Europe, d'Islam et d'ailleurs

\section{Prière d'insérer}

Marc Aymes, Jeanne Hersant et Élise Massicard

\section{(2) OpenEdition}

\section{Journals}

Édition électronique

URL : http://journals.openedition.org/labyrinthe/898

DOI : 10.4000/labyrinthe.898

ISSN : 1950-6031

Éditeur

Hermann

Édition imprimée

Date de publication : 22 juin 2005

Pagination : 13-19

Référence électronique

Marc Aymes, Jeanne Hersant et Élise Massicard, «Prière d'insérer », Labyrinthe [En ligne], 21 | 2005 (2), mis en ligne le 30 janvier 2009, consulté le 01 mai 2019. URL : http://journals.openedition.org/ labyrinthe/898; DOI : 10.4000/labyrinthe.898 


\section{PRIÈRE D'INSÉRER}

Marc Aymes, Jeanne Hersant, Élise MASSICARD

Qu'est-ce qu'une «communauté»? Partons du constat que ce terme est éminemment polysémique. D'un point de vue étique, il s'agit d'une construction intellectuelle inscrite dans plusieurs traditions sociologiques, et qui a parfois pu être constituée en idéal-type d'une organisation sociale, ce qui pose une question centrale: la communauté estelle un universel sociologique? Autrement dit, peut-elle décrire ou contribuer à comprendre n'importe quelle organisation sociale? $\leadsto$ «Rêver d'une société authentique»

\section{Étique/émique}

Le linguiste Kenneth Pike établit une opposition entre les points de vue étique (etic), mené à partir du point de vue propre au chercheur et à sa culture, et émique (emic) qui s'appuie sur les concepts et le système de pensée propres aux acteurs sociaux étudiés ${ }^{1}$.

En outre, ce terme est passé dans le sens commun, et est désormais employé autant par les journalistes que dans les discours quotidiens, contribuant à forger les représentations. Ainsi, tout groupe (surtout religieux) en migration ou, plus généralement, en situation «minoritaire», est presque spontanément qualifié de «communauté» (la «communauté musulmane», la «communauté juive de France»). Ces usages sont bien sûr corrélés aux contextes sociaux et politiques.

Enfin, ce terme peut recouvrir un statut susceptible d'être revendiqué et contesté, quand des droits collectifs sont reconnus à des groupes, et notamment dans des contextes dits multiculturalistes :

Le multiculturalisme ne signifie pas simplement la pluralité numérique de différentes cultures, mais plutôt une communauté qui crée, garantit, encou- 


\section{Labyrinthe, $n^{\circ} 21$}

rage des espaces dans lesquels différentes communautés peuvent se développer à leur rythme. Dans le même temps, il implique de créer un espace public dans lequel ces communautés peuvent interagir, enrichir la culture existante et créer une nouvelle culture consensuelle dans laquelle elles peuvent reconnaître des reflets de leur propre identité2.

En ce sens, l'emploi du terme «communauté» peut également être à usage politique.

Les difficultés posées par les usages multiples du terme « communauté » peuvent être illustrées de manière démonstrative à travers un cas où il est particulièrement délicat à manier, celui des alévis de Turquie. On regroupe sous le terme «alévi» des groupes hétérodoxes caractérisés par certaines pratiques cultuelles (adoration d'Ali, cérémonies spécifiques...) souvent qualifiées de syncrétiques ${ }^{3}$. Mais les frontières de cet ensemble restent floues, ce qui est renforcé par sa diversité interne, à la fois sur le plan linguistique - il existe des alévis turcophones, kurdophones, arabophones -, religieux et social. En outre, il n'est pas sûr que les alévis se perçoivent, ou soient perçus, comme une communauté. Le caractère problématique et la non-objectivation particulièrement marquée de cette hypothétique «communauté »-si tant est qu'il existe des communautés objectivées - posent la question de la pertinence, d'un point de vue scientifique, de qualifier les alévis de «communauté». Et ce d'autant plus que ce terme est souvent chargé de sens politique, puisqu'un mouvement identitaire - que nous appellerons aléviste - revendique une reconnaissance collective. Pour ce faire, ses cadres qualifient parfois les alévis de communauté - ou plutôt de divers termes indigènes dont le sens se rapproche de celui de notre «communauté », parfois non... Quels sont les enjeux de ces dénominations? Par ailleurs, les alévis n'ont aucun statut collectif en Turquie, mais ont récemment acquis celui de Religionsgemeinschaft (communauté religieuse) dans certains Länder d'Allemagne où ils se sont implantés suite à la migration. Ici se dessine une interrogation supplémentaire : en quoi la qualification ou l'auto-qualification comme «communauté » dépendent-elles du contexte, des institutions et des statuts existants?

2. Bikhu Parekh, Homi K. Bhabha, «Identities on Parade», Marxism today, juin 1989, p. 24-29.

3. Krisztina Kehl-Bodrogi, Die Kızlba?/Aleviten. Untersuchungen über eine esoterische Glaubensgemeinschaft in Anatolien, Berlin, Klaus Schwarz, 1988. 
Le chercheur doit-il alors parler de communauté? Et si oui, dans quelles conditions, et avec quelles précautions?

Bref, quels enjeux politiques et épistémologiques le terme et ses usages en contextes soulèvent-ils? Pour répondre à cette question, il faut distinguer entre les emplois propres aux savants et ceux propres aux acteurs, étique(s) et émique(s) de la «communauté», mais aussi s'interroger sur leurs liens et interactions ${ }^{4}$, car ils sont loin d'être imperméables. Cela implique également d'analyser les processus d'attribution et de revendication en contextes. Ces clarifications faites, l'usage de ce terme permet-il de mieux cerner la réalité sociale ?

E. M.

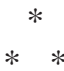

Autre point de départ : «Reconnaître qu'en effet il y a de la communauté entre passé et présent, mais que communauté - ce mot délicat n'est pas l'unique description possible de ces relations entre passé et présent $^{5}$.» D'où l'enjeu : la notion de «communauté » a quelque chose à voir avec les continuités que l'historien reconnaît dans l'histoire qu'il écrit.

Pour qui fait porter son regard sur l'actuel Orient méditerranéen - anciennement Empire ottoman -, cet enjeu se trouve investi d'une acuité particulière. Ainsi, s'agissant de l'histoire de la Terre sainte,

l'historiographie de la Palestine est dominée par les discours nationalistes des deux côtés du fossé israélo-palestinien, et [...] ces discours sont construits à partir de l'hypothèse d'une nette discontinuité avec le passé, introduite par l'intervention extérieure $[\ldots]^{6}$.

L'histoire nationaliste est sans continuités. Soit elle décrète une permanence absolue, l'identité d'une essence à elle-même : la nation,

4. Méthode utilisée notamment par Gerd Bauman, Contesting Culture : Discourses of Identity in Multiethnic London. Cambridge Studies in Social and Cultural Anthropology, New York and Cambridge, Cambridge University Press, 1996.

5. Raymond Williams, Keywords. A Vocabulary of Culture and Society, Londres, Fontana Press, 1988, p. 23.

6. Beshara Doumani, Rediscovering Palestine. Merchants and Peasants in Jabal Nablus, 1700-1900, Berkeley, University of California Press, 1995, p. XI. 
dira-t-on, a toujours existé, elle est l'essence incorruptible de l'Histoire, et l'historien rend simplement compte de ses variations phénoménales. Soit elle pose l'hypothèse d'une discontinuité radicale. S'en départir signifie, alors, la recherche de continuités à restaurer. Ce que propose, concernant la Palestine toujours, Ilan Pappé :

Élargir la vision historiographique consacrée sur la Palestine au cours de la fin du $\mathrm{x}^{\mathrm{e}} \mathrm{x}^{\mathrm{e}}$ siècle et du $\mathrm{xx}^{\mathrm{e}}$ siècle, une vision qui jusqu'à présent tend à être de nature conflictuelle, car principalement centrée sur le choc entre le sionisme et la population palestinienne locale. Nous souhaitons considérer l'histoire sociale de la Palestine comme un continuum au sein de l'histoire sociale de l'Empire ottoman - une perspective que les historiens n'ont commencé à adopter que récemment ${ }^{7}$.

Cet enjeu de continuités à trouver (ou à retrouver) engage, de manière redoublée, des histoires de communauté : car, du Proche-Orient arabe aux Balkans, l'histoire des États et des sociétés d'aujourd'hui est articulée, de manière privilégiée, à celle des «communautés» religieuses et des antagonismes qu'elles ont suscités - la progressive cristallisation des États-nations contemporains étant lue comme le terme logique de séculaires affrontements intercommunautaires.

De la Méditerranée d'hier à l'Europe d'aujourd'hui ou de demain, il convient d'interroger les traces laissées par ces communautés. Quelles trajectoires impriment-elles à l'histoire de «l'Orient compliqué»? Et quels lieux de savoir circonscrivent-elles ainsi?

Le terrain de l'enquête privilégié par ma contribution est, on l'aura compris, l'histoire de l'Empire ottoman à l'époque contemporaine (le $\mathrm{XIX}^{\mathrm{e}}$ siècle principalement) ${ }^{8}$. Un cas en particulier retient mon attention, en ce qu'il entrecroise plusieurs des dynamiques et problématiques explorées dans ce dossier: il s'agit de Chypre. S'y retrouvent en effet, tout à la fois, la complexité méconnue d'un passé ottoman, jusqu'au passage de l'île sous tutelle britannique en 1878; la hantise d'un « temps présent » marqué au coin de haines intercommunautaires ;

7. «The "Politics of Notables" to the "Politics of Nationalism": the Husayni Family, 1840-1922», dans Ilan Pappé, Moshe Ma'oz, (dir.), Middle Eastern Politics and Ideas : a History from Within, Londres/New York, Tauris Academic Studies, 1997, p. 163 (je souligne).

8. Se reporter à la carte p. 18 . 
et l'espoir (pour partie réalisé) d'un futur au sein de l'Union européenne, celle-là même qui, un temps, se fit appeler « communauté».

M. A.

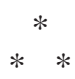

Il est question dans ce dossier de l'interprétation contemporaine des « communautés » du monde ottoman en termes de communauté nationale, et de la question des minorités dans le contexte turc post-ottoman; ma contribution se propose d'interroger les liens entre «minorité», «communauté » et «groupe ethnique » dans un contexte post-ottoman non turc, à savoir la Grèce, où une minorité fut constituée par un traité international lui reconnaissant des institutions propres et autonomes au sein de l'État-nation.

La Thrace occidentale (Thraki en grec, Batı Trakya en turc) est la région frontalière entre la Grèce, la Turquie et la Bulgarie. De province ottomane, elle est devenue possession bulgare à l'issue des guerres balkaniques, puis entité sous administration interalliée au lendemain de la Première Guerre mondiale ${ }^{9}$, avant d'être rattachée à la Grèce par le traité de Sèvres (1920). Hormis la période d'occupation bulgare à la faveur de l'occupation allemande de la Grèce, entre 1940 et 1944, la région est depuis lors restée grecque, avec néanmoins des spécificités institutionnelles liées au maintien sur place, en 1924, de la population musulmane locale, alors que tous les musulmans de Grèce étaient expulsés vers la Turquie selon les termes de l'échange de population énoncés dans le traité de Lausanne de 1923.

Un protocole additionnel à ce traité fut signé quelques mois plus tard entre la Grèce, la Turquie et des États «garants» (dont la France et la Grande-Bretagne), réglementant de façon symétrique le statut des minorités «musulmane» en Thrace occidentale ${ }^{10}$ et «grecque» à Istanbul (et

\footnotetext{
9. Joëlle Dalègre, « La mission du général Charpy en Thrace occidentale », Mesogeios 4 (1999), p. 66-76. 10. Cette minorité rassemble trois groupes : Pomaques, Tziganes et Turcs, qui se distinguent pas la langue parlée (le pomaque est un dialecte du bulgare, exclusivement oral) ou par le mode de vie: en effet, les Tziganes sont turcophones, mais «par extension, le terme tzigane se réfère rarement à la langue ou à la religion, mais plutôt à un niveau de vie qui se distingue de l'ensemble, et caractérise des personnes qui se trouvent au bas de la hiérarchie sociale». D'après Nikos Marantzidis et Georges Mavromatis, « Identité ethnique et comportement politique: le comportement électoral d'un groupe de Tziganes musulmans de Thrace occidentale (Grèce)», Études helléniques 8 (1), 2000, p. 71.
} 
dans les îles de Gökçeada et Bozcaada) ; le maintien d'une population en Thrace occidentale était d'ailleurs la contrepartie de celle des «Grecs orthodoxes » et du patriarcat œcuménique d'Istanbul. Seul le critère confessionnel fut retenu pour définir les populations concernées - la religion étant associée à un marqueur national -, mais l'actuelle république de Turquie n'ayant été proclamée que plusieurs mois après la signature du traité, seule une des deux minorités fut désignée par un adjectif renvoyant à une nationalité (" grecque ») tandis que, de l'autre côté, on se contentait d'un qualificatif religieux («musulmane »).

Le traité de Lausanne énonce, outre des droits équivalents à ceux dont jouissent les ressortissants des États respectifs, une série de droits positifs pour les deux minorités dites «non échangées». En ce qui concerne les «musulmans» en Thrace occidentale, il s'agit de la juridiction civile et religieuse des muftis (dignitaires religieux ayant autorité sur le personnel officiant dans les mosquées, les imams qui prêchent et les muezzins qui appellent à la prière), et d'un système d'enseignement mixte géré conjointement par les représentants de l'État grec et par ceux de la minorité.

Ces dispositions sont sans précédent en droit international : le traité de Lausanne a entériné l'acception contemporaine du terme «minorité», et a en plus prévu concrètement les modalités d'organisation de ces minorités au sein de la société majoritaire. Il a introduit du même coup le paradoxe suivant : alors que, au lendemain de la Première Guerre mondiale, le démantèlement des Empires austro-hongrois et ottoman devait marquer l'avènement de l'État-nation, deux situations minoritaires étaient définies en référence à l'organisation sociale et religieuse qui prévalait dans l'Empire ottoman, et structurées, à travers leurs institutions représentatives, sur un mode communautaire.

L'exemple de la Thrace occidentale vise à montrer la constitution d'une population distincte - schématiquement de l'extérieur et de l'intérieur - qui soit identifiable en tant que communauté, afin d'une part d'en faciliter la gestion, de l'autre de crédibiliser et de renforcer les revendications des acteurs politiques issus de cette population. Pour autant, penser ce phénomène en termes de stratégie (étatique pour l'extérieur, politique ou notabiliaire pour l'intérieur) ne suffit pas pour comprendre les interactions, et la façon dont les membres de cette minorité s'approprient le devoir de marquer les frontières du groupe, en perpétuant interdits et pratiques sociales distinctives. 


\section{Prière d'insérer}

Ainsi la confrontation des termes «minorité », «communauté » et «groupe ethnique» permet d'une part de mener une réflexion sur le pouvoir de nommer dont jouissent certains acteurs (le chercheur devenant éventuellement un acteur impliqué dans ce processus) et l'effet de réel de cette catégorisation; dès lors, les structures et dynamiques sociales aussi bien que les représentations en vigueur, ou «cadres de perception», deviennent intelligibles.

J. H. 